\title{
Development of geo-electrical meter based on networking*
}

\author{
WANG Lan-wei ${ }^{1), \uparrow}$ (王兰炜) ZHAO Jia-liu ${ }^{2)}$ (赵家骝) \\ 1) Institute of Crustal Dynamics, China Earthquake Administration, Beijing 100085, China \\ 2) Institute of Earthquake Science, China Earthquake Administration, Beijing 100036, China
}

\begin{abstract}
Further development of earthquake equipments is closely associated with that of computer technology. Because Embedded PC104 module has the equivalent functions of PC, it has been widely used in recent years, and can provide a new and flexible hardware design environment, but its applications in observation instruments of earthquake precursor are rare. The present paper introduces in detail the realization of a networked geo-electrical meter by applying the low price, high reliability embedded PC104 industrial computer.
\end{abstract}

Key words: network; embedded PC104 module; geo-electrical meter; data communication CLC number: P319.3 Document code: A

\section{Introduction}

Observation instruments of earthquake precursor have been replaced by digitalized instruments during the period of the Ninth Five-year Plan, resulting in significantly improvement of the observed data, both in quality and quantity, and the capability of earthquake monitoring in China. However, due to the burst of data, the conventional network based on telephone lines can no more satisfy the demands of data communication. Nowadays, with the development of computer, communication and network technology, the Internet, characterized by high speed, easy access and large capacity, has become a trend all over the world. Therefore, it is inevitable to reconstruct the earthquake observation network in our country taking full advantage of the Internet. This demands that the earthquake observation instruments must have the function of network communication based on Ethernet interface, enabling the monitoring command transmission and data exchange between remote computer and observation instrument can be carried out by the Internet.

\section{Functions of networked instrument}

As an independent node in a communication network, networked instrument should have the basic functions of data acquisition, remote control, auto alarm and data download. Users can get observation data and information via web browser, FTP client or other applications. Networked instrument can maximize their capability and users can easily control it in remote, which has changed the only local mode.

\footnotetext{
* Received 2008-03-24; accepted in revised form 2008-07-22.

Foundation item: "The Study of ELF Receiver" from Ministry of Science and Technology (2001BA601B03-01-03).

^Author for correspondence: wanglw829@126.com
} 
Networked instrument should have at least two functions. One is networked data communication and the other is remote control. The former includes response to data transmission commands, web service and FTP service, and the latter includes response to control commands (such as instrument's test, auto calibration, reset and reboot, etc.) and to set the instrument's parameters in remote. These functions will enhance the observation instruments to a higher technical level.

\section{The realization methods of networked instrument}

There are three main methods, based respectively on single-chip-microcomputers (SCM), special embedded system (SES) and universal embedded system (UES), for realizing a networked instrument.

\subsection{Based on SCM}

Generally, for the networked instruments based on SCM, an 8- or 16-bit microprocessor with TCP/IP protocol is adopted. The microprocessor has high integrity and abundant control functions, but it has a small capacity storage, limited resource and insufficiency functions.

At present, although TCP/IP protocol development kit can be provided by many SCM manufactures, this demands that the SCM has high executive speed and large-capacity program storage and a system designer masters the TCP/IP protocol and interface. Therefore, the realization of networked instruments based on SCM requires a very complicated and heavy workload on design of software and hardware. Meanwhile because of the limited resources of the SCM, it is difficult to expand the functions of the instrument.

\subsection{Based on SES}

The core of the SES is a 32-bit processor which has enough capability and resources to realize the required network protocol. Network connection can be implemented easily by the processor, but the hardware and OS system must be pruned according to a specific purpose.

However, it demands the users to be very familiar with the OS system of the processor while pruning. Otherwise very long time will be spent on development work and maybe end up with an unstable OS system. As a result, the overall system becomes unstable.

\subsection{Based on UES}

The UES (Take PC104 as representative) is an embedded industrial computer module which is completely compatible with standard computers and characterized by higher integrity, they are compact and small size $(90 \mathrm{~mm} \times 96 \mathrm{~mm})$, low power consumption, formed with a mature standard architecture, and with high reliability due to special design and manufacture. Compared with other systems, the UES apply more plentiful, cheaper and completely compatible software, hardware, peripheral and development tools with the PC, and accordingly enjoy more extensive technical support and system maintenance service. Therefore, they are more easily to be expanded, updated and serialized. However, they have the disadvantages of high cost and high power consumption.

For the design of the networked geo-electrical meter (NGM), a PC104 industrial computer is adopted as the control core which also enables the design of the original geo-electrical meter does not need to be changed. Not only the PC104's abundant resource can be used to realize data acquisition, data storage, system control and the network functions of the instrument through simple software design, but also the functions of the NGM can be improved and expanded without requiring complex work on the basis of the original geo-electrical meter. 


\section{Realization of the NGM}

\subsection{System design of NGM}

At present, the model of geo-electrical meter used in field is a ZD8B geo-electrical meter with a RS-232 serial port. The meter receives commands from and exchanges data with the computer only via its RS-232 serial port. In order to realize the network functions of the geo-electrical meter by the simplest way, a high performance PC104 industrial computer is connected to the ZD8B via its serial port to develop the NGM. In order to maintain the original hardware design, software and work mode of ZD8B unchanged, the measurement process and data acquisition are still controlled by the SCM while network communication, data acquisition system monitoring, web service and FTP service is performed by the PC104 computer. Figure 1 illustrates the basic design of the NGM.

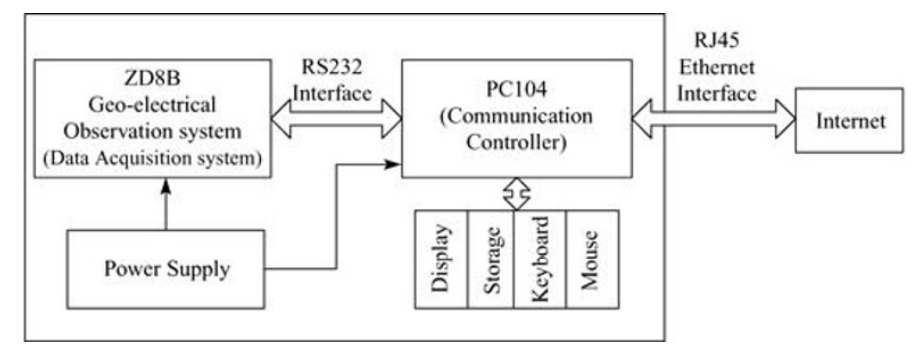

Figure 1 The basic design of the NGM

In this system, PC104 is multi-functional.

1) Communicator

(a) Receiving data from data acquisition system via the serial port, control commands and data transmission command, and responds, from remote computer via the Internet.

(b) Web service

(c) FTP service

2) Controller

Controls the data acquisition system via the serial port, such as data acquisition parameter setting, system clock adjustment, system test, calibration and zeroing.

3) Processor

Processes the acquired data and accomplishes higher resolution results.

4) Operator

Providing a more friendly and clearer operation interface

\subsection{Selection of OS system}

The PC104 industrial computer is based on the PC architecture, which is adaptive to all popular OS system, enabling to choose among different kinds of OS systems.

Although DOS is the most direct, simplest and clearest single task OS system, it can't satisfy the demands of multiple tasks.

Windows is the most popular multiple task real-time OS system. It not only has the advantages of stability and reliability, but also can manage all kinds of interface, peripheral and system memory, execute multiple tasks and support graphic function. In addition, it has a simple user interface and an easy program. Accordingly, Windows has been widely used in development of such instruments. 
The geo-electrical meter observes and records data every one hour. In the meantime, it responds to network controlling command, web request and data upload or download. This is a simple multiple task system. Therefore, it is reasonable to choose the simplified version Windows 98 as OS system, which not only has the advantages of stability and reliability, but supports multiple tasks and uses fewer system resources.

\section{Design of software}

\subsection{Selection of program language}

Winsocket control of Visual Basic development environment is adopted as the program tool. At present, most network communication programs are programmed by using Socket (WANG and ZHONG et al, 2002). Winsocket is a standard network communication interface of Windows system, and adopts $\mathrm{C} / \mathrm{S}$ architecture to enable data exchange between customer and server (TANG and TONG, et al, 2002).

Visual Basic development environment can satisfy our demands because of its friendly user interface, easy program and shorter development work time.

\subsection{Functions and flowcharts of the application program}

\subsubsection{Communication functions}

1) Network communication

In the entire observation network, the mode of communication between remote computer and the NGM in observation station is the typical C/S architecture. The NGM is taken as a server for listening to a specified port all the time. After the NGM receives a request of connection and accepts it, the connection between the remote computer and the NGM is set up. Then data communication can be carried out, such as data transmission, observation parameter setting and state information transmission.

The functions of startup listening and port monitoring are as follows.

$\begin{array}{ll}\text { Winsock1(0).LocalPort=ServerPort } & \text { ' Network port } \\ \text { Winsock1(0).Listen } & \text { 'Start port listening } \\ \text { Winsock1(0).State } & \text { 'Port state monitoring }\end{array}$

Accepting a request and setup connection:

Winsock1(WhichSocket).Accept requested 'Accept connect request

2) Serial Communication

Communication controller receives data from the measurement unit and sends control command to it via the serial port.

Opening the serial port and setting communication parameter are accomplished by the following functions.

Public sub serialport_open()

MSComm1.CommPort=1

'Set port number

MSComm1.RThreshold $=1$

'Set receiving byte

MSComm1.InBufferCount $=0$

'Clear receiving buffer

MSComm1.InBufferSize $=1024$

'Set the size of receiving buffer

MSComm1.InputLen $=0$

'Receive all the data in buffer

MSComm1.InputMode $=$ comInputModeBinary 'Set the receiving mode as binary

MSComm1.Settings="2400,n,8,1" ' Set port baud rate

MSComm1.PortOpen=True 'Open port 
End sub

\subsubsection{WEB Service}

HTTP protocol is adaptive to the communication between the web server and browser which is used by customer to communicate with the web server. When the web server receives a request from user, it processes the request and returns the demanded data (XU and CHAI, et al, 2002).

As a web server, the NGM performs the following functions.

1) Data upload/download: Storage of up to 30 days' data which can be downloaded by browser.

2) Remote real-time control: Enabling users to control the observation system through browser, such as starting measurement, setting up and calibrating system time.

3) Parameter setting: After the user's request is received by the NGM, parameters can be set up. Parameters include operation and state parameters such as initiation values, observation parameter.

4) System test: Enabling the user to test the NGM through browser, including zero and full scale calibration, leakage current test, HL voltage measurement.

5) Visit level setting and authority: The visit level and authority are set to 3 levels. As long as the user's authority is not lower than the demand of visit level and the user has been authorized by the server, he can operate the NGM within his authority range. The first level is for the ordinary users who can only browse the web. The second level is for the senior user who can upload/download data, modify system parameter and remotely control the system. The third level is for the administrator who can do everything.

\subsubsection{FTP Service}

As a FTP server, the NGM provides data download/upload. After authorized, remote users can download/upload the observation data.

FTP program is programmed using Visual $\mathrm{C}++$ language. The login function is as follows.

The function of checking username and password:

CUser user;

If(theServer.m_UserManager.CheckUser(m_strUserName, strArguments, user))

\{

m_strCurrentDir = "/";// set home directory of user

m_bLoggedon $=$ TRUE;

//Correct username and password, successfully logged on

SendResponse("230 Logged on");

else

//Incorrect username or password, return information

SendResponse("530 Login or password incorrect!");

\subsection{The Program interface and Flowcharts}

The NGM is composed of a data acquisition module and the main control module. The main function of data acquisition module is measuring apparent resistivity and transferring the result to main control module. The function of the main control module is storing, processing, displaying and transmitting data on the network. Figure 2 shows the main operation interface of the NGM. Observation data is displayed in the middle part. The status bar at the bottom displays communication state, time, etc. The menu at the top is used for operating the NGM, such as parameter setting, data transmission, system test and data view. Among these, parameter setting is used to set up 
network parameters, system time, sampling parameters and so on in order to make NGM work properly. Data transmission is used to receive yesterday's data, today's data and data acquisition module's parameter from data acquisition unit. System test is used to send all kinds of command to data acquisition module and check system's state. Data view is used to display data in curve or text pattern.

The interface of NGM is direct and simple which can enable users to monitor and control the system's state easily. Figure 3 shows the curve of apparent resistivity at Hefei station from June 1 to December 31, 2007.

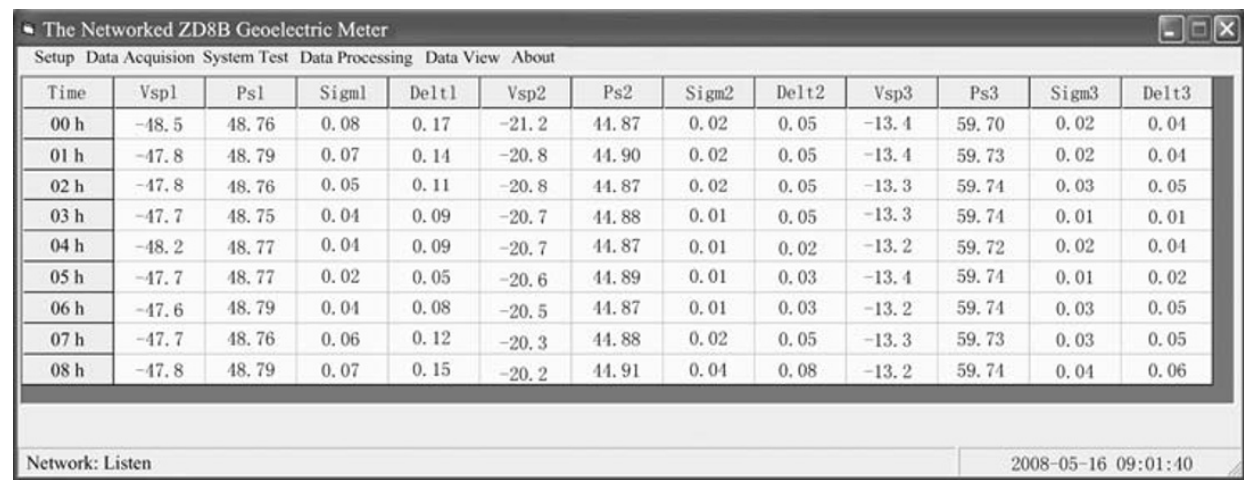

Figure 2 The operating interface of NGM

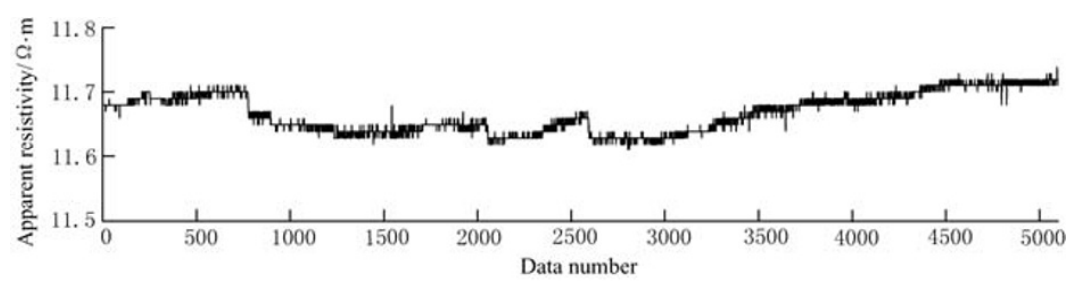

Figure 3 The curve of apparent resistivity at Hefei station from June 1 to December 31, 2007

\section{Summary}

Compared with serial communication, networked instrument has the main advantages of rapid response to command, high data transmission speed and high reliability. It can use the rich resources of the Internet and share data in wider range. Therefore networked instrument has become a trend.

Winsocket program technology has been used in the communication software during the course of the development of NGM. The software has very high communication reliability. At present, NGM has been used in the project of "China Digital Earthquake Observatory Network". During last two years, the observation result manifests that NGM has very high reliability and all network functions. Now these instruments are running very good.

Although only the realization of NGM is discussed in the present paper, because of the high integrity, high reliability and easy expansion, especially the versatility of $\mathrm{PC} 104$, the realization method of NGM can be used in the implementation of any earthquake observation instrument with a serial port. 


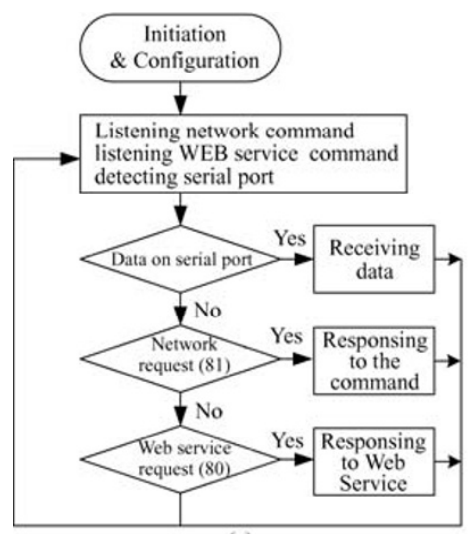

(a)

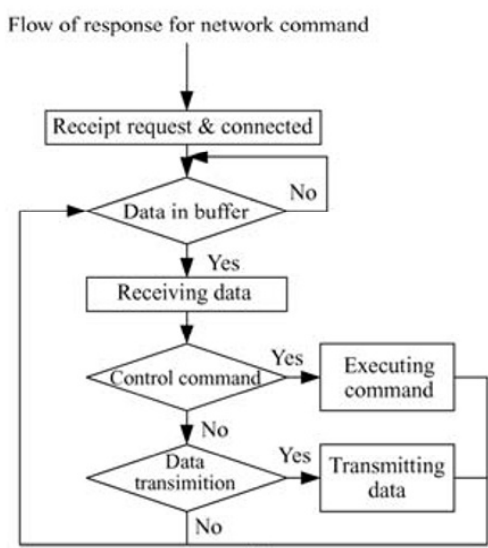

(b)

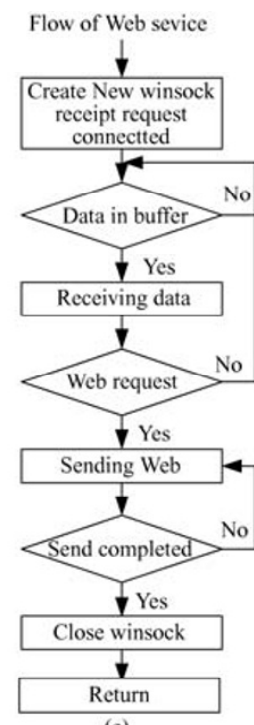

(c)

Figure 4 The flowchart of the program

(a) The flowchart of monitor program; (b) The flowchart of communication program; (c) The flowchart of web sever program

\section{References}

TANG Zhi and TONG Lu-yuan. 2002. Application of WinSock in communication of multimedia remote supervisory control system [J]. Electric Power Automation Equipment, 22(5): 29-31 (in Chinese).

WANG Xiao-ping and ZHONG Jun. 2003. Visual Basic Network Communications Protocol Analysis and Application [M]. Beijing: Post and Telecommunications Press: 1-13 (in Chinese).

XU ming and CHAI Zhi-lei. 2000. An implement of embedded web server based on TCP/IP Sockets [J] Journal of East China Shipbuilding Institute (Natural Science Edition), 15(5): 39-41 (in Chinese). 